\title{
The effect of intra-articular vanilloid receptor agonists on pain behavior measures in a murine model of acute monoarthritis
}

This article was published in the following Dove Press journal:

Journal of Pain Research

17 August 2016

Number of times this article has been viewed

\author{
Mishal Abdullah' \\ Maren L Mahowald ${ }^{2}$ \\ Sandra P Frizelle ${ }^{2}$ \\ Christopher W Dorman ${ }^{2}$ \\ Sonia C Funkenbusch ${ }^{2}$ \\ Hollis E Krug ${ }^{2,3}$ \\ 'Department of Medicine, \\ Rheumatology Fellowship Training \\ Program, University of Minnesota \\ Medical School, ${ }^{2}$ Department of \\ Medicine, Minneapolis Veterans' Affairs \\ Health Care System, ${ }^{3}$ Department \\ of Medicine, University of Minnesota \\ Medical School, Minneapolis, MN, USA
}

Correspondence: Hollis E Krug Rheumatology Office (I I IR), Minneapolis Veterans' Affairs Medical Center, I Veterans Drive, Minneapolis, MN 554I7, USA

Tel +l 6I24674I98

Email hollis.krug@va.gov

\begin{abstract}
Arthritis is the most common cause of disability in the US, and the primary manifestation of arthritis is joint pain that leads to progressive physical limitation, disability, morbidity, and increased health care utilization. Capsaicin (CAP) is a vanilloid agonist that causes substance $\mathrm{P}$ depletion by interacting with vanilloid receptor transient receptor potential V1 on small unmyelinated $\mathrm{C}$ fibers. It has been used topically for analgesia in osteoarthritis with variable success. Resiniferatoxin (RTX) is an ultra potent CAP analog. The aim of this study was to measure the analgesic effects of intra-articular (IA) administration of CAP and RTX in experimental acute inflammatory arthritis in mice. Evoked pain score (EPS) and a dynamic weight bearing (DWB) device were used to measure nociceptive behaviors in a murine model of acute inflammatory monoarthritis. A total of 56 C57B16 male mice underwent EPS and DWB testing - 24 nonarthritic controls and 32 mice with carrageenan-induced arthritis. The effects of pretreatment with $0.1 \%$ CAP, $0.0003 \%$ RTX, or $0.001 \%$ RTX were measured. Nociception was reproducibly demonstrated by increased EPS and reduced DWB measures in the affected limb of arthritic mice. Pretreatment with $0.001 \%$ RTX resulted in statistically significant improvement in EPS and DWB measures when compared with those observed in carrageenan-induced arthritis animals. Pretreatment with IA $0.0003 \%$ RTX and IA $0.01 \%$ CAP resulted in improvement in some but not all of these measures. The remaining 24 mice underwent evaluation following treatment with $0.1 \%$ CAP, $0.0003 \%$ RTX, or $0.001 \%$ RTX, and the results obtained were similar to that of naïve, nonarthritic mice.
\end{abstract}

Keywords: pain, resiniferatoxin, capsaicin, vanilloid, intra-articular

\section{Introduction}

Arthritis is the most common cause of disability in the US. It is estimated that $>50$ million adults (one out of every five people) suffer from some form of arthritis. ${ }^{1}$ One of the main goals of arthritis management is pain reduction. Currently, available oral systemic analgesics are limited by insufficient joint pain relief, intolerable drug side effects, and adverse drug interactions. ${ }^{2,3}$

Other agents for intra-articular (IA) administration, such as corticosteroids, hyaluronic acid derivatives, and botulinum toxin $\mathrm{A}$, have variable analgesic effects and duration of action. ${ }^{4-7}$ Therefore, there is still an unmet need for new therapies for refractory arthritis pain.

Murine models of joint nociception are commonly used in preclinical studies of new analgesic therapies. IA carrageenan (CAR) has been employed to induce a murine model of nociception in acute monoarticular inflammatory arthritis. ${ }^{7,8}$ Measures of evoked 
pain thresholds and tenderness have been successfully used to quantify nociception in mouse models of arthritis. ${ }^{8}$ Analysis of dynamic weight bearing (DWB) measures has been found to be extremely sensitive for quantifying limb nociception severity. ${ }^{9,10}$

Capsaicin (CAP) and resiniferatoxin (RTX) are vanilloid agonists that have attracted much attention due to their effect on alleviating different kinds of pain. ${ }^{11} \mathrm{CAP}$ is a vanilloid agonist that exerts its analgesic effects by binding to transient receptor potential V1 (TRPV1) channels in sensory nerves. ${ }^{12}$ These toxins are nonselective cation ionophores involved in integration of afferent noxious signals generated by mediators of inflammation. CAP triggers a calcium influx by binding to TRPV1 channels, followed by generation of an action potential and depletion of substance $P$ making the neuron unresponsive to additional painful stimuli. ${ }^{13}$ Other potential mechanisms include the effects of sustained high levels of intracellular calcium that may activate calcium-dependent enzyme proteases ${ }^{14}$ or induce depolymerization of cytoskeletal components such as microtubules. ${ }^{15,16}$ CAP has been used as a topical analgesic for arthritis pain. ${ }^{17}$

RTX is a CAP analog derived from latex of Euphorbium resinifera, which is a potent agonist of TRPV $1 .{ }^{18}$ In contrast to CAP, Jeffry et al ${ }^{18}$ demonstrated that low concentrations of RTX caused a slow and sustained depolarization of membrane potential, thereby preventing the generation of action potentials.

The aim of this study was to determine whether IA administration of CAP or RTX would produce an analgesic effect in experimental acute inflammatory arthritis joint nociception. We compared the evoked and spontaneous nociceptive responses of male C57Bl6 mice before and after IA injection of CAR, which produced severe, acute inflammatory monoarthritis. We then measured the nociceptive pain behaviors in arthritic mice with and without pretreatment with CAP and two doses of RTX.

\section{Materials and methods}

\section{Animal subjects}

A total of 56 male C57B16 mice from the Jackson Laboratory (Bar Harbor, ME, USA) aged 11-12 weeks were studied. Animals were housed in groups of eight in the Animal Care and Research Facility at the Minneapolis Veterans Affairs Medical Center, a facility approved by the Association for Assessment and Accreditation of Laboratory Animal Care International. The animals were acclimated to the vivarium for 1 week before experimentation and were housed in standard polycarbonate $183 / 4 \times 101 / 4 \times 81 / 4$ in $^{3}$ cages with water and standard rodent diet ad libitum. Environmental conditions were maintained at $72^{\circ} \mathrm{F} \pm 1^{\circ} \mathrm{F}$ and $33 \% \pm 1 \%$ humidity, with 12-hour light/dark cycles. All animal procedures and protocols were approved by the Minneapolis Veterans Administration Health Care System (VAHCS) Institutional Animal Care and Use Committee and conformed to the "Guide for the Care and use of Laboratory Animals". ${ }^{19}$

\section{Study design}

Seven groups of eight animals each (56 animals) were studied. Four groups of eight animals (32 animals) were studied, prior to any IA injections. Eight animals subsequently received IA CAR to produce acute inflammatory arthritis nociception and were examined 3 hours after IA injection of CAR when nociceptive responses peak.

Three vanilloid treatment groups of eight mice each (24 animals) were pretreated with IA vanilloids (0.01\% CAP, $0.001 \%$ RTX, or $0.0003 \%$ RTX) 7 days prior to induction of arthritis and examined 3 hours following IA CAR. Three groups (24 animals) of nonarthritic mice received equivalent doses of IA vanilloids and served as nonarthritic controls for the treatment groups (Figure 1).

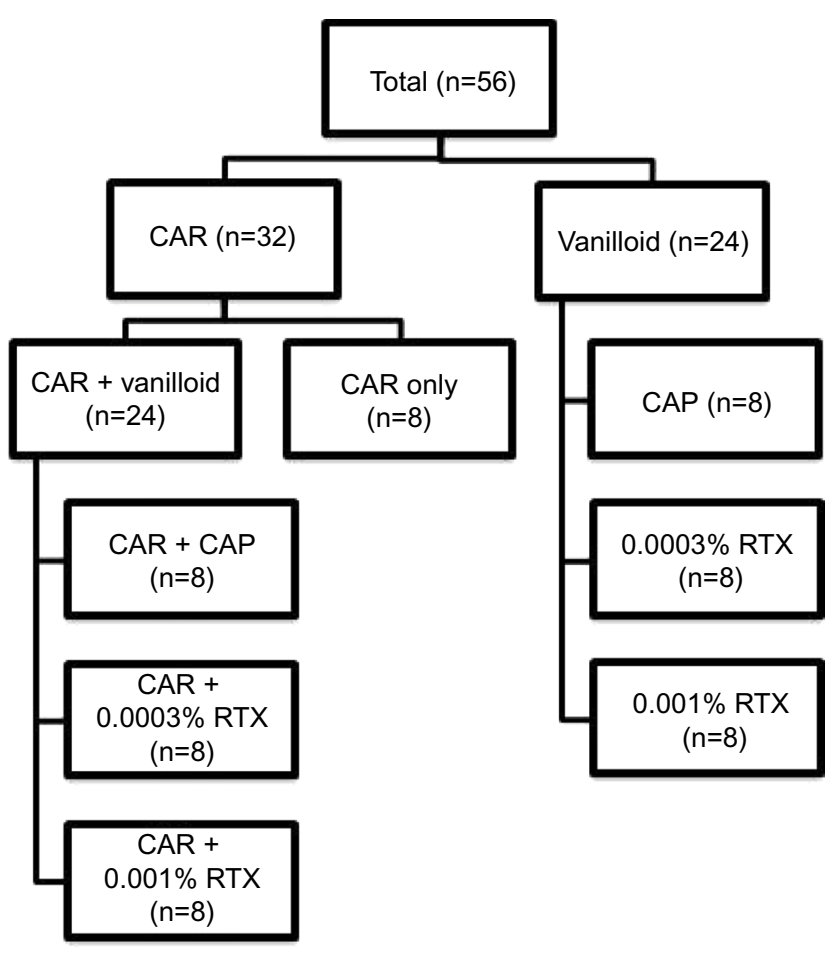

Figure I Overview of study.

Notes: A total of 56 animals were studied in seven groups of eight mice each. Thirty-two naïve, nonarthritic animals were studied obtaining EPS, DWB measures for weight, and DWB measures for time. The same 32 animals were then reevaluated for the same parameters 3 hours after arthritis induction in the left hind limb by IA CAR injection. Of this group, 24 were also pretreated with IA vanilloid (eight pretreated with CAP, eight pretreated with $0.0003 \%$ RTX, and eight pretreated with $0.001 \%$ RTX). Twenty-four additional mice were studied for the same parameters after IA vanilloid administration alone without arthritis induction.

Abbreviations: EPS, evoked pain score; DWB, dynamic weight bearing; IA, intraarticular; CAR, carrageenan; CAP, capsaicin; RTX, resiniferatoxin. 


\section{Joint injections}

Injections were performed into the left knee through the infrapatellar ligament using a $30 \mathrm{G}$ needle with a customized sheath that limited needle penetration to $2.5 \mathrm{~mm}$. The mice were anesthetized with isoflurane in oxygen (induction and maintenance $3 \%, 1 \mathrm{~L} / \mathrm{min}$ ) for $3 \pm 1$ minutes during the injections. The left knee was shaved and the skin disinfected with $70 \%$ ethanol solution. CAP and RTX groups were injected 7 days prior to examination with $10 \mathrm{~mL}$ of either 0.01\% CAP (MP Biomedicals, Sigma Aldrich Co., St Louis, MO, USA\#202757), 0.0003\% RTX (Sigma-Aldrich Co., St Louis, MO, USA, \#R8756), or $0.001 \%$ RTX diluted in $0.9 \%$ sterile saline. The CAR groups were injected 3 hours prior to examination with $10 \mathrm{~mL}$ of $2.5 \%$ CAR (Sigma-Aldrich Co., \#22049-5G-F) diluted in 0.9\% sterile saline. Animals recovered under a heat lamp until normal ambulation was observed.

\section{Measures of nociceptive behavior}

Both evoked and spontaneous nociceptive pain behaviors were measured. Evoked pain score (EPS) was measured as the total number of fights and vocalizations during 1 minute of firm palpation of the knee at a frequency of $1 / \mathrm{s} .{ }^{8}$ The control right knee was examined before the left knee for comparison. Examinations were performed by a single-blinded examiner trained in consistent manual restraint methods and trained with a Palpometer (Palpometer Systems, Inc., Victoria, BC, Canada) to deliver a series of repeated firm palpations of $\sim 1,100 \mathrm{gf} / \mathrm{cm}^{2}$ or $15.6 \mathrm{psi}$.

Spontaneous nociceptive behavior was measured as the offloading of body weight (measured as a percentage of total body weight) from the painful limb and reduced time (measured as a percentage of total time in the device) on the painful limb using the DWB device (Bioseb, Vitrolles, France). To increase the sensitivity of detecting spontaneous nociceptive behaviors, each mouse was placed in the DWB device after measurement of EPS. The device is a $4.5 \times 4.5 \times 8.25 \mathrm{in}^{3}$ plexiglass cube with a floor consisting of 1,936 pressure sensors. The device software calculated the percentage of time spent and percentage of weight placed on each of the four limbs during a 5-minute period. We compared the percentage of time spent and the percentage of weight placed on each of the two hind limbs.

\section{Statistical analysis}

EPS and ADWB for the five independent groups (naïve, CAR, CAP, HD RTX, and LD RTX) were compared using a one-way analysis of variance. Dunnett's post hoc $t$-test for comparing each of the other four groups with the CAR group was used whenever the overall analysis of variance was significant.

\section{Results}

Characterization of nociceptive behaviors produced in the murine model of CAR-induced acute knee arthritis

EPSs from the knees of 32 naïve animals were $<2$ and equivalent bilaterally ( $\mathrm{SD}=1.58$ in the left and $\mathrm{SD}=0.97$ in the right). IA CAR-induced arthritis resulted in increased EPSs in the left knee (mean $=6.25$ fights and/or vocalizations per mouse, $S D=3.0$; Figure 2) and a difference between left and right knee EPSs was 6.125 (SD =3.18). Spontaneous nociceptive behaviors measured with the DWB device demonstrated equal weight bearing on each hind limb of $40 \%$ in naïve nonarthritic animals and a significant decrease to $30 \%$ in the left hind limb with CAR-induced arthritis $(P<0.05$, SD $=8.9)$. The difference between left and right weight bearing increased from $<1 \%$ to $17 \%(P<0.0005, \mathrm{SD}=11.7)$. The average time spent on each hind limb was equivalent in naïve nonarthritic mice. Time spent on the left hind limb decreased from $96 \%$ to $88 \%$ with CAR-induced arthritis in the left knee $(P<0.05, \mathrm{SD}=9.3)$ and the difference between the left and right hind limbs increased from $<1 \%$ in naïve mice to $11 \%$ in the arthritic mice $(P<0.0001, \mathrm{SD}=9.87$; Figure 3$)$.

\section{Measuring the analgesic effects of IA vanilloids in CAR-induced acute knee arthritis}

Groups of eight mice each underwent pretreatment with $0.01 \%$ CAP, $0.0003 \%$ RTX, or $0.001 \%$ RTX prior to arthritis induction. IA CAP pretreatment reduced the EPS score by $48 \%$ from 6.25 to 3.25 ( $P<0.026$; Figure 2$)$. The DWB measures for weight increased from $30 \%$ to $36 \%$ in the CAP-treated arthritic mice $(P=0.16)$ when compared with the untreated arthritic mice. The time on the arthritic hind limb increased from $88 \%$ in arthritic untreated mice to $97 \%$ in the CAP-treated mice - the normal amount ( $P=0.003$; Figure 3$)$.

IA $0.001 \%$ RTX (high dose) pretreatment significantly reduced the EPS from 6.25 to 1.5 (a near normal score; $P<0.0001$; Figure 2). DWB measure of percentage of weight on the arthritic limb increased from $30 \%$ to $38.5 \%(P=0.024)$ and time on rear limbs significantly increased from $88 \%$ to 97\% $(P<0.004$; Figure 3$)$.

Pretreatment with the lower dose $0.0003 \%$ RTX also reduced the EPS significantly from 6 to $1.5(P<0.0001$; Figure 2). The average weight on hind limbs only increased from $30 \%$ to $33 \%$ not significant (NS) but time on hind limbs increased from $88 \%$ to $95 \%$ ( $P=0.033$; Figure 3$)$.

Each vanilloid treatment significantly reduced the right to left knee difference in EPSs (Figure 4). 


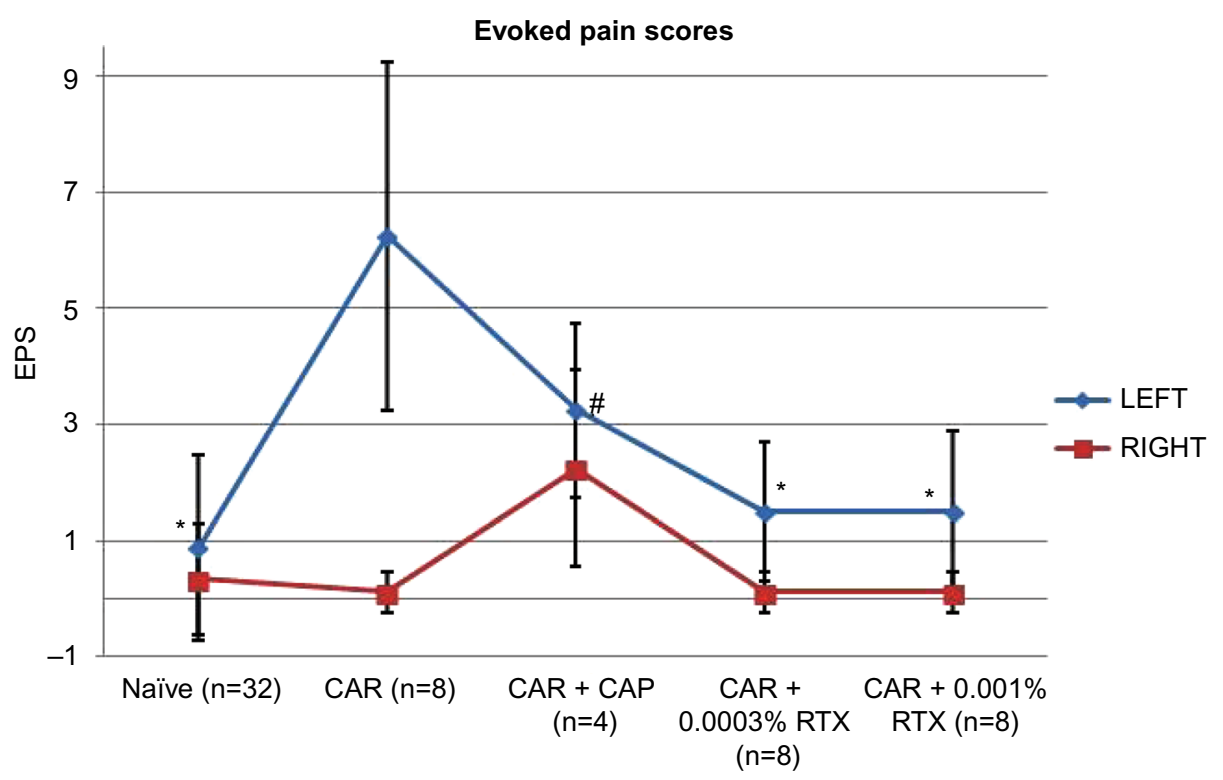

Figure 2 Evoked pain scores.

Notes: EPS for right (red line) and left (blue line) hind limbs. Right hind limbs served as internal controls and did not undergo any IA injections. The left hind limb groups included the naïve nonarthritic group (naïve), arthritic (CAR), and arthritic pretreated group (CAR + CAP, CAR + 0.0003\% RTX, and CAR + 0.00I\% RTX). Following induction of arthritis with IA CAR, the mean EPS was elevated in the left hind limbs. Pretreatment with vanilloid prior to arthritis induction resulted in relatively improved EPS in the left hind limbs when compared with the right. One-way analysis of variance comparing EPS for treatment groups in the left hind limb found a significant difference between groups $(F=15.6, P<0.000 \mathrm{I})$. Post hoc analysis using Dunnett's test to compare between group differences found that the $C A R$ group EPS was significantly higher than naïve $(P<.000 I)$ and when compared with all treatment groups $(P<0.05) .\left({ }^{*} P<0.0001, \# P<0.05\right)$.

Abbreviations: EPS, evoked pain score; CAR, carrageenan; CAP, capsaicin; RTX, resiniferatoxin; IA, intra-articular.

The left to right difference in percentage of time of weight bearing significantly decreased from $11.4 \%$ in arthritic mice to $<1 \%, 3 \%$, and $1 \%$ in treated animals (Figure 4 ).

Left to right differences in percentage of weight bearing decreased from 17.3 to 1.5 with CAP $(P=0.004)$, to 7.9 with low-dose RTX $(P=0.142)$, and to 7.5 with the high-dose RTX ( $P=0.118$; Figure 3$)$. Although the results for RTX were not quite statistically significant, there did seem to be a dose effect seen between low-dose $(0.0003 \%)$ and high-dose $(0.001 \%)$ RTX in percentage of weight bearing.

\section{Normal right knee controls}

Throughout all stages of this study, the contralateral right knee was a normal, nonarthritic internal control. After induction of arthritis, EPSs in the contralateral knee fell slightly from a baseline mean of $0.34(\mathrm{SD}=0.0 .97)$ to $0.125(\mathrm{SD}=0.35)$, $P=$ NS. The DWB measures for mean weight and time of the nonarthritic right hind limb were analyzed for each separate group, and these values failed to show any statistically significant difference when compared with the naïve, nonarthritic mice except for an increase in percentage of weight bearing of the right hind limb in the arthritic group (46.89\%, SEM $1.68)$ and the high-dose RTX group (46.0\%, SEM 1.56) compared with the naïve group (41.2\%, SEM 1.18), $P<0.05$ (Figure $3 \mathrm{~A}$ ) and a decrease in right limb weight bearing in the CAP-pretreated arthritic group (37.35\%, SEM 1.51) compared with the arthritic group $(P<0.001)$. This appears to indicate compensation by the nonarthritic right hind limb in the face of nociception in the arthritic left hind limb that is reversed when the nociception is reduced in the arthritic limb.

\section{IA vanilloid agonist control}

IA vanilloid agonist treatment in naïve, nonarthritic mice did not result in significant change in EPS or DWB measures when compared with data obtained from naïve, nonarthritic mice (Figure 5).

\section{Discussion}

This study demonstrated that IA CAR-induced acute inflammatory knee arthritis produced measurable evoked and spontaneous nociceptive behaviors that were used to measure analgesic effects of IA injections of vanilloid agonists.

IA CAR induced an acute inflammatory monoarthritis with peak intensity at 3-4 hours similar to that of previous studies..$^{20,21}$ EPSs increased 3 hours after IA CAR injections. The spontaneous nociceptive behaviors measured as changes in DWB were related to offloading from the painful limb with compensatory increased loading of the normal limb. Weight bearing then improved with IA analgesia. This study confirms that a shift of body weight from the painful 
A

DWB weight
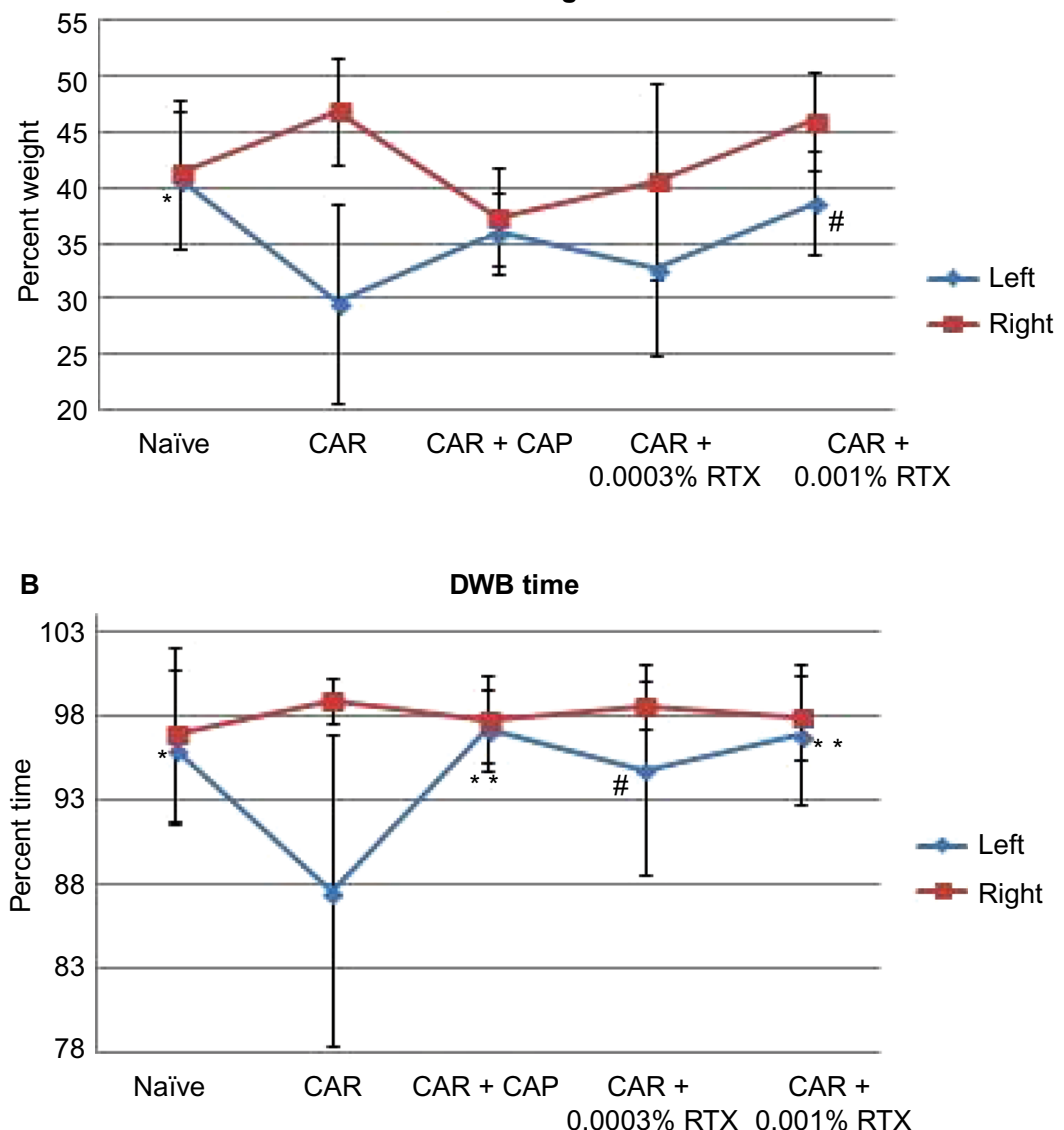

Figure 3 Dynamic weight bearing measures.

Notes: Mean $( \pm S D)$ DWB measures for weight and time in left and right hind limbs for different groups of mice. (A) DWB measures for weight and (B) time in left and right hind limbs; right hind limbs (red line) were internal controls and did not undergo IA injections. Left hind limbs (blue line) were measured in different groups including naïve, nonarthritic animal (naïve), arthritic animal (CAR), and arthritic animals pretreated with vanilloids (CAR + CAP, CAR + 0.0003\% RTX, and CAR + 0.001\% RTX). One-way analysis of variance for effect of treatment groups on weight bearing of the left hind limb showed a significant effect $(F=6.38, P=0.0002)$. Post hoc analysis comparing all groups

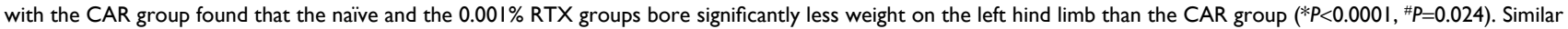
analysis of \% time on the limb also showed a significant effect for the treatment group ( $F=4.71, P=0.0023)$. Post hoc analysis demonstrated a more consistent treatment effect across all treatments with significant differences between the naïve group and all treatment groups compared with $C A R$. $* P=0.00 I, \# P=0.033$, and $* * P<0.005$.

Abbreviations: DWB, dynamic weight bearing; IA, intra-articular; CAR, carrageenan; CAP, capsaicin; RTX, resiniferatoxin.

arthritic limb to the contralateral normal limb reflects a weight-bearing deficit and is a reliable measure of spontaneous nociception in models of knee joint arthritis. ${ }^{12,13,22,23}$ These results were similar to a study of CAR arthritis in rats and our previous results using IA botulinum toxin in mice. $^{8,17}$

We showed that pretreatment with IA CAP and IA RTX normalized some pain behavior measures. Both vanilloids normalized DWB measures for time spent on the arthritic limb. These improvements were statistically significant. Differential weight bearing deficits tend to improve but the change was statistically significant only in the group pretreated with high-dose RTX compared with the arthritic left hind limbs. Interestingly, even though vanilloid treatment did not normalize weight bearing, in the left hind limbs compared with arthritic left hind limbs, differences between right and left hind limbs disappeared with CAP pretreatment and were reduced with all doses of RTX pretreatment. Quadrupeds may compensate for hind limb pain with fore limb weight bearing. In some cases, this symmetric offloading of the hind limbs seemed to be compensated for by increased loading of the forelimbs, but this was not consistent.

Although there was improvement in pain behaviors with IA vanilloid receptor agonists, this improvement was often incomplete and variable. We controlled for the effect of IA injection so close to the time of examination. IA injection alone had no effect on nociceptive pain measures. Previous studies have demonstrated that IA treatment with saline does not improve EPS in experimental acute inflammatory monoarthritis. ${ }^{8}$ Therefore, it is not likely that IA injection alone or the vehicle used for the specific treatments had any effect. We were concerned that irritants such as RTX and CAP could 


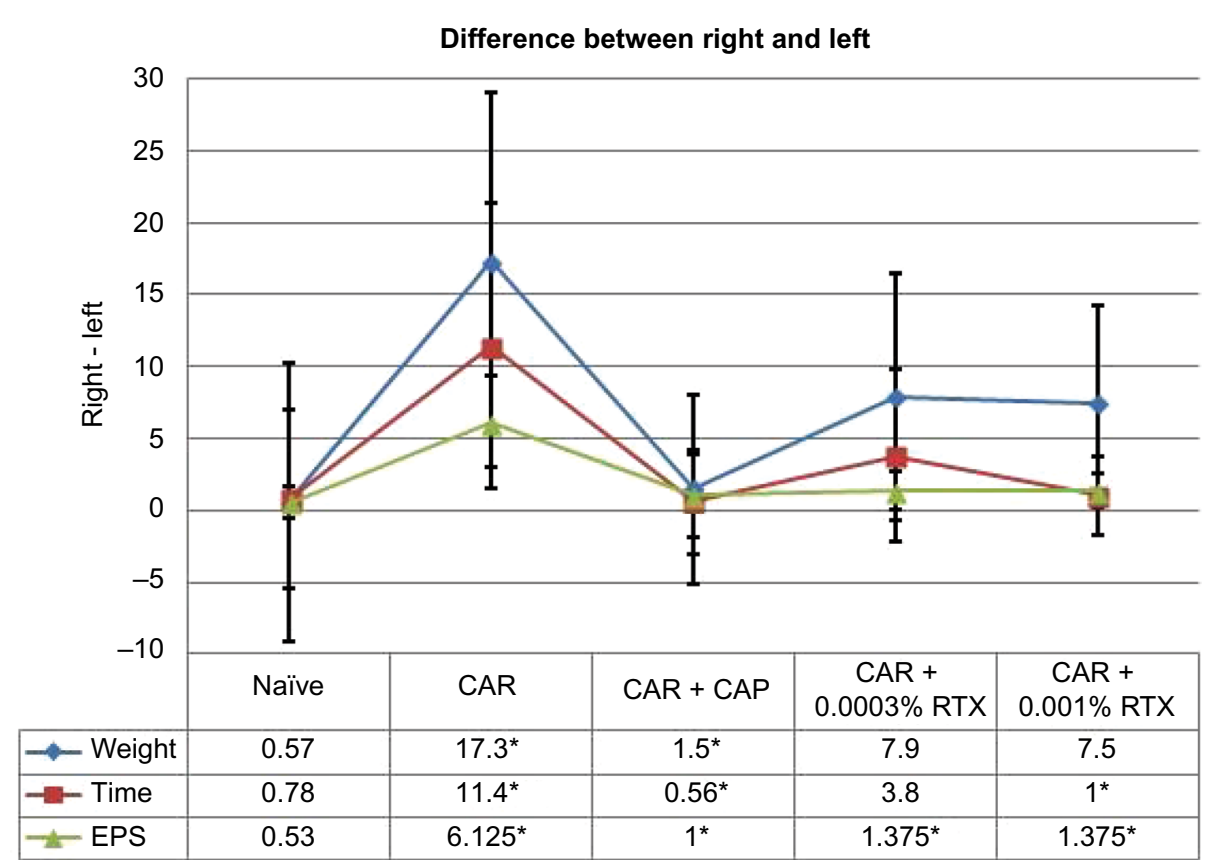

Figure 4 Difference in right and left hind limb nociceptive measures.

Notes: Difference between left and right hind limb DWB measures for weight (blue line), time (red line), and EPS (green line) in different experimental groups including naïve, arthritic (CAR), and arthritic pretreated with vanilloid (CAR + CAP, CAR $+0.0003 \%$ RTX, and CAR + 0.00I\% RTX). Changes in left and right hind limb differences were a more sensitive way to assess for change in response to interventions including arthritis induction and pretreatment with vanilloid. One-way analysis of variance for group effect was significant for all three measures. Post hoc analysis compared CAR with naïe and with all treatment groups (CAP, $0.0003 \% \mathrm{RTX}$, and $0.001 \% \mathrm{RTX}$; $* P<0.05)$. Abbreviations: DWB, dynamic weight bearing; EPS, evoked pain score; CAR, carrageenan; CAP, capsaicin; RTX, resiniferatoxin.

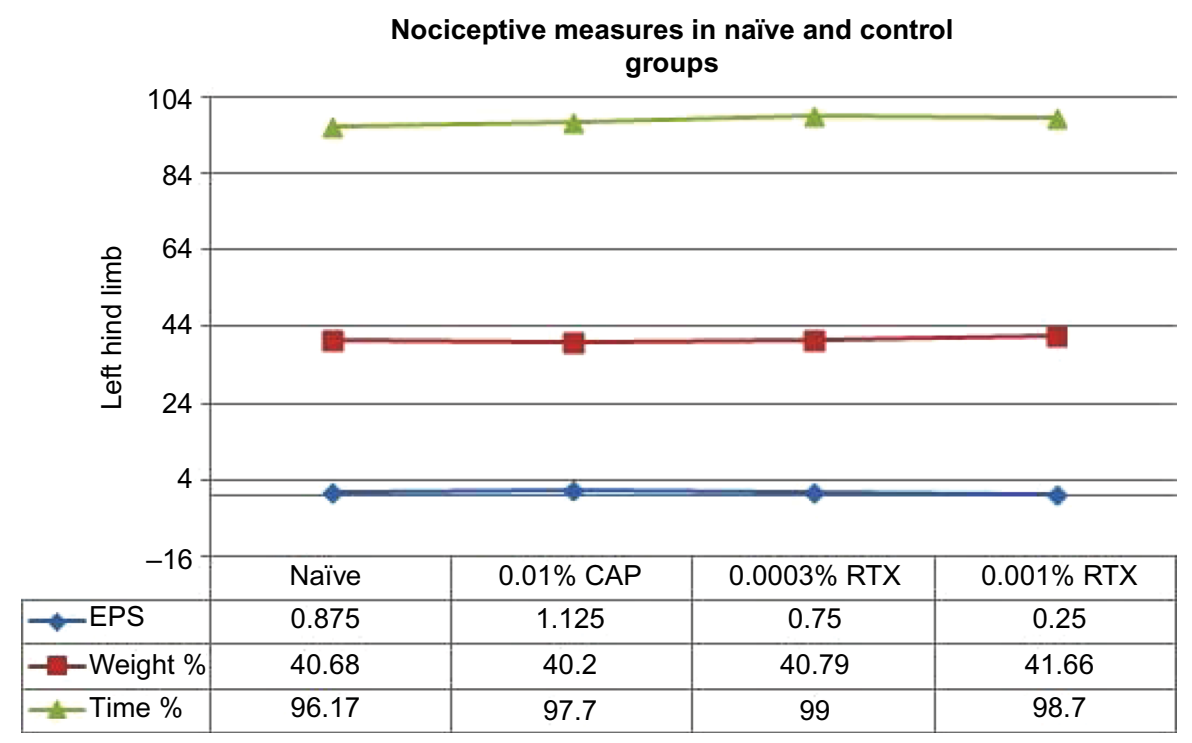

Figure 5 Nociceptive measures (EPS, DWB weight\%, and DWB time\%) for naïve animals and vanilloid agonist controls.

Note: Nociceptive measures including EPSs, DWB weight\%, and DWB time\% of left knees from the vanilloid agonist control groups show similar results to the results from the naïve, nonarthritic controls.

Abbreviations: EPS, evoked pain score; DWB, dynamic weight bearing; CAP, capsaicin; RTX, resiniferatoxin.

produce a nociceptive response in the absence of joint pain, but IA injection of vanilloid alone did not produce a change in nociceptive pain behaviors.

The high-dose IA RTX injection was often more effective suggesting a dose effect. This higher dose is equal to $0.1 \mathrm{mg}$ and is significantly lower than previous locally administered doses. $^{24,25}$

RTX seemed to provide more reliable analgesia than CAP. RTX is considered to be less of an irritant than CAP and the mechanism of action is different. Although they share a 
vanillyl group that binds to TRPV1, CAP binds to vanilloidsensitive peptidergic neurons causing a rapid release of SP, whereas RTX causes a slow and sustained depolarization of the membrane and also binds to serum proteins. This low affinity protein binding may affect the pharmacokinetics of this compound producing a slower onset and more sustained response. ${ }^{18,26,27}$

The typical time of onset of vanilloid-induced analgesia and the short time of onset of CAP-induced nociception require that the vanilloids should be given prior to the induction of joint pain in this model. It will be important to determine whether IA vanilloid treatment can be effective in chronic arthritis pain when given after the joint pain has been established. Certainly, topical treatments have been shown to be effective, ${ }^{17}$ but the results presented here support further studies of these two vanilloids as potential IA analgesics in humans as has been previously suggested. ${ }^{28}$

Our study has several potential limitations. The groups were small so that real differences may not have been detected. The results cannot be extrapolated to other models of experimental arthritis nociception because the experiments were conducted only in mice with acute CAR-induced monoarthritis. In these murine studies, as well as in previous similar studies, the IA procedures were carried out under short duration general anesthesia. This may not be necessary or practical in humans. An alternative would be to administer RTX or CAP mixed with local anesthetic. Time-dose studies with the toxins are needed to optimize toxin dose and dosing intervals. The duration of analgesic effects is not known and would be difficult to establish in this model because the acute arthritis nociception only lasts 3-4 hours. Future studies are planned to examine the effect of IA vanilloids in mouse models of chronic joint pain.

\section{Conclusion}

Our experiment showed that the IA administration of the vanilloid agents, CAP and RTX, in mice was safe and produced analgesia in an experimentally induced acute inflammatory arthritis pain model. It may have the potential for use in humans with refractory chronic arthritis pain.

\section{Acknowledgments}

Many thanks to Thomas Rector, $\mathrm{PhD}$, who was very helpful in designing and performing the statistical analysis. This work was supported by Merit Review Award \# RX000379-05 from the US Department of Veterans Affairs Rehabilitation Research and Development Service. The contents do not represent the views of the US Department of Veterans Affairs or the United States Government.

\section{Disclosure}

The authors report no conflicts of interest in this work.

\section{References}

1. Centers for Disease Control and Prevention. Prevalence of doctordiagnosed arthritis and arthritis-attributable activity limitation United States, 2007 - 2009. MMWR Morb Mortal Wkly Rep. 2010;59(39): 1261-1265.

2. Mahowald M. The Role of Nonprescription Analgesics in Treating Mild to Moderate Pain. Clinical and Economic Considerations. Minneapolis: Minneapolis Health Learning Systems; 2000.

3. Mahowald ML. Chronic pain management. In: Ruddy S, Harris E, Sledge C, Budd R, Sergent J, editors. Kelley's Textbook of Rheumatology. Philadelphia, PA: WB Saunders; 2004:7th Edition, p967-995

4. Bellamy N, Campbell J, Welch V, Gee TL, Wells GA. Intraarticular corticosteroid for treatment of osteoarthritis of the knee. Cochrane Database Syst Rev. 2006;(2):CD005328.

5. Cheng J, Abdi S. Complications of joint, tendon and muscle injections. Tech Reg Anesth Pain Manag. 2007;11(3):141-147.

6. Bannuru RR, Natov NS, Obadan IE, Price LL, Schmid CH, McAlindon TE Therapeutic trajectory of hyaluronic acid versus corticosteroids in the treatment of knee osteoarthritis: a systematic review and meta-analysis. Arthritis Rheum. 2009;61(12):1704-1711.

7. Mahowald ML, Krug HE, Singh JA, Dykstra D. Intra-articular botulinum toxin type A: a new approach to treat arthritis joint pain. Toxicon. 2009;54(5):658-667.

8. Krug HE, Frizelle SP, McGarraugh P, Mahowald ML. Pain behavior measures to quantitate joint pain and response to neurotoxin treatment in murine models of arthritis. Pain Med. 2009;10(7):1218-1228.

9. Yu YC, Koo ST, Kim CH, Lyu Y, Grady JJ, Chung JM. Two variables that can be used as pain indices in experimental animal models of arthritis. J Neurosci Methods. 2002;115(1):107-113.

10. Tetreault P, Dansereau M-A, Dore-Savard L, Beaudet N, Sarret P. Weight bearing evaluation in inflammatory, neuropathic and cancer chronic pain in freely moving rats. Physiol Behav. 2011;104(3):495-502.

11. Szallasi A, Blumberg PM. Vanilloid receptors: new insights enhance potential as a therapeutic target. Pain. 1996;68(2-3):195-208.

12. Caterina MJ, Julius D. The vanilloid receptor: a molecular gateway to the pain pathology. Annu Rev Neurosci. 2001;24:487-517.

13. Jessell TM, Iversen LL, Cuello AC. Capsaicin-induced depletion of substance P from primary sensory neurons. Brain Res. 1978;152(1): 183-188.

14. Chard PS, Bleakman D, Savidge JR, Miller RJ. Capsaicin-induced neurotoxicity in cultured dorsal root ganglion neurons: involvement of calcium-activated proteases. Neuroscience. 1995;65(4): 1099-1108.

15. Goswami C, Schmidt H, Hucho F. TRPV1 at nerve endings regulates growth cone morphology and movement through cytoskeleton reorganization. FEBS J. 2007;274(3):760-772.

16. Han P, McDonald HA, Bianchi BR, et al. Capsaicin causes protein synthesis inhibition and microtubule disassembly throughTRPV1 activities both on the plasma membrane and intracellular membranes. Biochem Pharmacol. 2007;73(10):1635-1645.

17. Kissin EY, Freitas CF, Kissin I. Effects of intraarticular resiniferatoxin in experimental knee-joint arthritis. Anesth Analg. 2005;101(5): 1433-1439.

18. Jeffry JA, Yu S-Q, Sikand P, Parihar A, Evans MS, Premkumar LS. Selective targeting of TRPV1 expressing sensory nerve terminals in the spinal cord for long lasting analgesia. PLoS One. 2009;4(9): e7021. 
19. The National Academies Press. Guide for the Care and Use of Laboratory Animals. 8th ed. Washington, DC: The National Academies Press; 2011.

20. Min SS, Han JS, Kim YI, et al. A novel method for convenient assessment of arthritic pain in voluntarily walking rats. Neurosci Lett. 2001; 308(2):95-98.

21. Tonussi CR, Ferreira SH. Rat knee-joint carrageenin incapacitation test: an objective screen for central and peripheral analgesics. Pain. 1992;48(3):421-427.

22. Pomonis JD, Boulet JM, Gottshall SL, et al. Development and pharmacological characterization of a rat model of osteoarthritis pain. Pain. 2005;114(3):339-346.

23. Otsuki T, Nakahama H, Niizuma H, Suzuki J. Evaluation of the analgesic effects of capsaicin using a new rat model for tonic pain. Brain Res. 1986;365(2):235-240.
24. Menendez L, Juarez L, Garcia E, Garcia-Suarez O, Hidalgo A, Baamonde A. Analgesic effects of capsazepine and resiniferatoxin on bone cancer pain in mice. Neurosci Lett. 2006;393(1):70-73.

25. Xu XJ, Farkas-Szallasi T, Lundberg JM, Hokfelt T, Wiesenfeld-Hallin Z, Szallasi A. Effects of the capsaicin analogue resiniferatoxin on spinal nociceptive mechanisms in the rat: behavioral, electrophysiological, and in situ hybridization studies. Brain Res. 1997;752(1-2):52-60.

26. Szolcsanyi J, Szallasi A, Szallasi Z, Joo F, Blumberg PM. Resiniferatoxin: an ultrapotent selective modulator of capsaicin-sensitive primary afferent neurons. J Pharmacol Exp Ther. 1990;255(2):923-928.

27. Holzer P. Capsaicin: cellular targets, mechanism of action and selectivity for thin sensory neurons. Pharmacol Rev. 1991;43(2):143-201.

28. Szallasi A, Blumberg PM. Vanilloid (capsaicin) receptors and mechanisms. Pharmacol Rev. 1995;51(2):159-212.

\section{Publish your work in this journal}

The Journal of Pain Research is an international, peer reviewed, open access, online journal that welcomes laboratory and clinical findings in the fields of pain research and the prevention and management of pain. Original research, reviews, symposium reports, hypothesis formation and commentaries are all considered for publication.

\section{Dovepress}

The manuscript management system is completely online and includes a very quick and fair peer-review system, which is all easy to use. Visit http://www.dovepress.com/testimonials.php to read real quotes from published authors. 